University of Wollongong

Research Online

Faculty of Engineering - Papers (Archive)

Faculty of Engineering and Information

Sciences

July 2005

\title{
Solid-state thermionics and thermoelectrics in the ballistic transport regime
}

T. E. Humphrey

University of Wollongong

Mark F. O'Dwyer

University of Wollongong, markod@uow.edu.au

C. Zhang

University of Wollongong, czhang@uow.edu.au

R. A. Lewis

University of Wollongong, roger@uow.edu.au

Follow this and additional works at: https://ro.uow.edu.au/engpapers

Part of the Engineering Commons

https://ro.uow.edu.au/engpapers/162

\section{Recommended Citation}

Humphrey, T. E.; O'Dwyer, Mark F.; Zhang, C.; and Lewis, R. A.: Solid-state thermionics and thermoelectrics in the ballistic transport regime 2005.

https://ro.uow.edu.au/engpapers/162

Research Online is the open access institutional repository for the University of Wollongong. For further information contact the UOW Library: research-pubs@uow.edu.au 


\title{
Solid-state thermionics and thermoelectrics in the ballistic transport regime
}

\author{
T. E. Humphrey, ${ }^{\text {a) }}$ M. F. O’Dwyer, C. Zhang, and R. A. Lewis \\ School of Engineering Physics, University of Wollongong, Wollongong, New South Wales (NSW) 2522, \\ Australia
}

(Received 3 February 2005; accepted 27 May 2005; published online 22 July 2005)

\begin{abstract}
It is shown that the equations for electrical current in solid-state thermionic and thermoelectric devices converge for devices with a width equal to the mean free path of electrons, yielding a common expression for the intensive electronic efficiency in the two types of devices. This result is used to demonstrate that the material parameters for thermionic and thermoelectric refrigerators are equal, rather than differing by a multiplicative factor as previously thought. (C) 2005 American Institute of Physics. [DOI: 10.1063/1.1977191]
\end{abstract}

Solid-state thermionic devices may be distinguished from thermoelectric devices according to whether electron transport is ballistic or diffusive. ${ }^{1}$ There is, however, little to distinguish the underlying thermodynamics of the two types of devices, with both achieving reversibility under the same conditions $s^{2,3}$ and both being governed by the same "material parameter." ${ }^{, 6}$ In a previous work, a proportionality factor of $F_{0} / F_{1 / 2} \sqrt{\pi}$, where $F_{n}$ is a Fermi integral, was found between the two material parameters. ${ }^{6}$

Here we show that the equations for diffusive and ballistic transport converge for devices with a width equal to the mean free path of electrons. This results in a common equation for the intensive electronic efficiency for both thermionic and thermoelectric devices, and a proportionality factor between their material parameters equal to unity. We explain the discrepancy between this and a previous work by pointing out an inconsistency in the energy dependency of the relaxation time in the two equations for conductivity used in Ref. 6.

Current density in both thermionic and thermoelectric devices can be expressed as

$$
J=\iiint j(\mathbf{k}) d \mathbf{k},
$$

where $j(\mathbf{k}) \delta \mathbf{k}$ is the net "energy-resolved" current of electrons flowing in the direction opposite to the temperature gradient with momentum in the range $\delta \mathbf{k}$ around $\mathbf{k}$. In thermionic devices with a width less than the mean free path, most electrons travel ballistically from one reservoir to another. In this case the energy-resolved current density is given by (with dependence upon $\mathbf{k}$ implicit)

$$
j^{b}(\mathbf{k}) \delta \mathbf{k}=q D_{r} \zeta v_{x}^{r} \Delta f_{0} \delta \mathbf{k},
$$

where $D_{r}(\mathbf{k})$ is the density of states (DOS) in the reservoirs, $\zeta(\mathbf{k})$ is the probability that electrons are transmitted between the reservoirs, $v_{x}^{r}(\mathbf{k})$ is the velocity in the direction of transport given by the dispersion relation $E(\mathbf{k})$ in the reservoirs, and $\Delta f_{0}=\left[f_{0}\left(\mathbf{k}, \mu_{C}, T_{C}\right)-f_{0}\left(\mathbf{k}, \mu_{H}, T_{H}\right)\right]$ is the difference in the Fermi occupation of states in the cold/hot reservoirs, where

\footnotetext{
${ }^{a)}$ Electronic mail: tammy.humphrey@unsw.edu.au
}

$$
f_{0}(\mathbf{k}, \mu, T)=\left[1+\exp \left(\frac{E(\mathbf{k})-\mu}{k T}\right)\right]^{-1}
$$

and where $\mu_{C / H}$ is the electrochemical potential and $T_{C / H}$ the temperature of electrons at the cold/hot ends of the device.

Here we follow a previous work, ${ }^{6}$ and assume the transmission probability depends upon the total momentum of the electrons rather than the momentum in the direction of transport only, allowing a direct comparison with thermoelectrics in which the energy of mobile electrons is also restricted in all three dimensions [this assumption is made implicitly in Ref. 6, between Eqs. (1.1) and (1.3)]. The theoretical differences between thermionic devices in which the transmission probability is a function of $\mathbf{k}$ and $k_{x}$ are explored in detail in other papers. $^{7-9}$

In thermoelectric devices the energy-resolved diffusive electron current density may be obtained from the Boltzmann transport equation under the relaxation-time approximation, and can be written as ${ }^{10}$

$$
j^{d}(\mathbf{k}) \delta \mathbf{k}=q D_{l} \tau\left(v_{x}^{l}\right)^{2} \frac{d f_{0}}{d x} \delta \mathbf{k},
$$

where $D_{l}(\mathbf{k})$ is the local DOS, $v_{x}^{l}$ is the velocity of electrons in the direction of transport, determined from the local dispersion relation $E(\mathbf{k})$, and $\tau(\mathbf{k})=\tau_{0} E(\mathbf{k})^{r}$ is the relaxation time in the direction of transport.

In solid-state power generators and refrigerators with a width close to the electronic mean free path, it is expected that Eqs. (2) and (4) should yield the same results. To show this, we take the energy dependence of the relaxation time to be $r=-1 / 2$, which corresponds to scattering that is dominated by acoustic phonons, and results in a mean free path in the direction of transport, $\lambda \equiv v_{x}(\mathbf{k}) \tau(\mathbf{k})$, which is independent of energy. ${ }^{6}$ We also note that $d f_{0}(x) / d x \approx\left[f_{0}(x)-f_{0}(x\right.$ $+\delta x)] / \delta x$ when $\delta x$ is small, so that, for a piece of thermoelectric material $L=\lambda$ in length, $d f_{0}(x) / d x \approx \Delta f_{0} / \lambda$ and Eq. (4) becomes

$$
j^{d}(\mathbf{k}) \delta \mathbf{k}=q D_{l} v_{x}^{l} \Delta f_{0} \delta \mathbf{k} .
$$

It can be seen that Eqs. (2) and (5) have the same form, where the product $\zeta(\mathbf{k}) D_{r}(\mathbf{k}) v_{x}(\mathbf{k})$ in Eq. (2) plays the same 
role in determining the energy spectrum of the electrons which carry current as the product $D_{r}(\mathbf{k}) v_{x}^{r}(\mathbf{k})$ does in Eq. (5). This simple result provides an additional underpinning for the observation of Ulrich et al. ${ }^{6}$ that thermionic and thermoelectric devices refrigerate (or generate power) via the same underlying physical mechanism. To show that there is no sharp transition in the behavior of a solid-state power generator or refrigerator as its width changes from $L<\lambda$ to $L>\lambda$, one can use the fact that the probability that an electron can travel a distance $L$ without suffering a collision is ${ }^{11}$

$$
P=\exp (-L / \lambda)
$$

to obtain an equation for the energy-resolved current density useful in solid-state power generators and refrigerators of length $L \approx \lambda$ as

$$
j(\mathbf{k}) \delta \mathbf{k}=q \Delta f_{0}\left\{\frac{\lambda}{L} v_{x}^{l} D_{l}[1-P]+v_{x}^{r} D_{r} \zeta P\right\} \delta \mathbf{k},
$$

which can easily be generalized to the case where $\lambda \rightarrow \lambda(\mathbf{k})$.

Equality between Eqs. (2) and (5) results in a common expression for the electronic efficiency ${ }^{12}$ (in thermionic devices) and intensive efficiency ${ }^{13,14}$ (across a small section of thermoelectric material) of

$$
\Phi_{\mathrm{PG}}^{b}=\left|V J / \dot{Q}_{H}\right|
$$

for a solid-state power generator and

$$
\Phi_{R}^{b}=\left|\dot{Q}_{C} / V J\right|
$$

for a solid-state refrigerator, where the heat flux density in the cold/hot reservoir of a thermionic device, or at the cold/ hot ends of the small section of a thermoelectric material, is given by

$$
\dot{Q}_{C / H}=\mp \iiint\left[E(\mathbf{k})-\mu_{C / H}\right] \frac{j(\mathbf{k})}{q} d \mathbf{k},
$$

where the $-/+$ refers to the cold/hot case, and it is assumed that the temperature gradient, electric field $(\varepsilon=V / L)$, and current have no components in the $y$ and $z$ dimensions. It is important to point out that Eq. (10) considers only heat carried by electrons. If phonon-mediated heat leaks are also considered, then two extra terms should be added to Eq. (10), the first being $\pm \Delta T \kappa_{l} / L$, where $\kappa_{l}$ is the thermal conductivity of the lattice, while the second accounts for the "Joule" heat released by electrons which diffuse between the hot and cold reservoirs, part of which is then carried by phonons to each end of the device. Shakouri et al. ${ }^{15}$ have shown that in the limit $L \gg \lambda$ then exactly half of this heat $V J$ is deposited in the hot and cold reservoirs while if $L \ll \lambda$, this heat is entirely deposited in the reservoir receiving the net current of electrons.

Finally, it can be shown that the above results yield the same expression for the material parameter in thermionic and thermoelectric refrigerators. Assuming a dispersion relation of $E=\hbar^{2} \mathbf{k}^{2} / 2 m^{*}$ and three-dimensional reservoirs so that $D$ $=1 /(2 \pi)^{3}$. Eq. (2) may be used to obtain the same thermionic materials parameter as found in Ref. 6, expressed in terms of the mean free path $\lambda$ as

$$
\beta_{\mathrm{TI}}=\frac{\lambda}{\kappa_{l}} \frac{4 \pi m^{*} k}{h^{3}}\left(k T_{C}\right)^{2} .
$$

The thermoelectric material parameter is given by ${ }^{6}$

$$
\beta=\frac{\sigma T}{\kappa_{l}} \frac{k^{2}}{q^{2}} F_{0}(\eta)^{-1},
$$

where $\sigma$ is the electrical conductivity and $F_{n}$ is a Fermi integral given by

$$
F_{n}(\eta)=\frac{1}{\Gamma(n+1)} \int_{0}^{\infty} \frac{\epsilon^{n}}{\exp (\epsilon-\eta)+1} d \epsilon,
$$

where the reduced Fermi energy is $\eta=\left(\mu-E^{\prime}\right) / k T_{C}$ and $E^{\prime}$ is the height of the barrier in thermionic devices (so that the transmission probability in Eq. (2) becomes $\zeta=0$ for $E<E^{\prime}$, $\zeta=1$ for $E>E^{\prime}$ ) or the conduction-band edge in a piece of thermoelectric material. Using the same dispersion relation and density of states as for thermionic devices, we can write an equation for conductivity in thermoelectric materials as

$$
\begin{aligned}
\sigma & \equiv \frac{q^{J^{d}}}{d \mu / d x}=\frac{4 \pi m^{*} q^{2} \lambda}{h^{3}} \int E \frac{d f_{0}(E)}{d E} d E \\
& =\lambda \frac{4 \pi m^{*} k}{h^{3}} q^{2} T F_{0}(\eta)
\end{aligned}
$$

where we have used the facts that $d f_{0} / d x=(d \mu / d x)$ $\times\left(d f_{0} / d \mu\right)$ for $d T / d x=0$, that $d f_{0} / d \mu=-d f_{0} / d E$, and have integrated by parts to obtain the final line. It can be seen by substitution that this yields $\beta=\beta_{\mathrm{TI}}$ if $T=T_{C}$.

In Ref. 6 the thermionic and thermoelectric materials parameters were expressed in terms of the mobility of electrons. In order to do this for the thermoelectric material parameter, two different expressions for conductivity were equated:

$$
\sigma=-q^{2} \int v_{x}^{2} \tau D_{l} \frac{d f_{0}}{d E} d E,
$$

where $\tau=\tau_{0} E^{-1 / 2}$, and

$$
\begin{aligned}
& \sigma=n q \mu=q \mu \int D_{l} f_{0} d E \\
& =-\frac{q^{2} \tau}{m^{*}} \int D_{l} E \frac{d f_{0}}{d E} .
\end{aligned}
$$

Given that $v_{x}^{2} \propto E$ and $\mu \equiv q \tau / m^{*}$, it can be seen that the two equations do not give the same result for conductivity as the relaxation time is assumed to be proportional to $E^{-1 / 2}$ in the first and independent of energy in the second, and this difficulty results in a factor of $F_{0} / F_{1 / 2} \sqrt{\pi}$ identified in Ref. 6 .

In summary, it has been shown that solid-state thermionic and thermoelectric devices with a width close to the mean free path of electrons share a common equation for electrical current density and electronic efficiency, and that their material parameters for refrigeration are equal. This result means that in the specific case where the product $\zeta(\mathbf{k}) D_{r}(\mathbf{k}) v_{x}(\mathbf{k})$ in a thermionic device is equal to the product $D_{l}(\mathbf{k}) v_{x}^{l}(\mathbf{k})$ in a thermoelectric device for all values of $\mathbf{k}$ 
which make a contribution to the current (above the barrier for thermionic device or above/below the conduction band in $n$ - $p$-type thermoelectric materials), then the performance of these two types of devices is expected to be indistinguishable in the regime where the device length is of the order of a mean free path.

\section{ACKNOWLEDGMENTS}

One of the authors (T.E.H.) is supported by a grant from the NSW Sustainable Energy Research and Development Fund (SERDF) and the Australian Research Council. Three of the authors (M.F.O'D., C.Z., and R.A.L.) are supported by the Australian Research Council. One of the authors (T.E.H.) acknowledges helpful discussions with A. Shakouri.

${ }^{1}$ G. S. Nolas, J. Sharp, and H. J. Goldsmid, Thermoelectrics Basic Principles and New Materials Developments (Springer, Berlin, 2001).

${ }^{2}$ T. E. Humphrey, R. Newbury, R. P. Taylor, and H. Linke, Phys. Rev. Lett. 89, 116801 (2002).
${ }^{3}$ T. E. Humphrey and H. Linke, Phys. Rev. Lett. 94, 096601 (2005).

${ }^{4}$ C. B. Vining and G. D. Mahan, J. Appl. Phys. 86, 6852 (1999).

${ }^{5}$ A. Shakouri and C. LaBounty, Proceedings of the 18th International Conference on Thermoelectrics, ICT99, Baltimore, MD, 1999 (IEEE, Piscataway, NJ, 1999), p. 35.

${ }^{6}$ M. D. Ulrich, P. A. Barnes, and C. B. Vining, J. Appl. Phys. 90, 1625 (2001).

${ }^{7}$ D. Vashaee and A. Shakouri, Phys. Rev. Lett. 92, 106103 (2004).

${ }^{8}$ M. F. O’Dwyer, T. E. Humphrey, R. A. Lewis, and C. Zhang, submitted to Phys. Rev. B; cond-mat/0506388.

${ }^{9}$ T. E. Humphrey, M. O’Dwyer, and H. Linke, J. Phys. D 38, 2051 (2005).

${ }^{10}$ C. Kittel, Introduction to Solid State Physics, 8th ed. (Wiley, New York, 2005).

${ }^{11}$ N. W. Ashcroft and N. D. Mermin, Solid State Physics (Saunders College Publishing, Orlando, Florida, 1976), p. 249.

${ }^{12}$ G. N. Hatsopoulos and E. P. Gyftopoulos, Thermionic Energy Conversion: Processes and Devices (MIT, Cambridge, Massachusetts, 1973), Vol. 1.

${ }^{13}$ C. Vining, Symposium on Thermoelectric Materials-New Directions and Approaches, edited by T. M. Tritt, MRS Symposia Proceedings No. 278 (Materials Research Society, Pittsburgh, 1997), p. 3.

${ }^{14}$ G. J. Snyder and T. S. Ursell, Phys. Rev. Lett. 91, 148301 (2003).

${ }^{15}$ A. Shakouri, E. Y. Lee, D. L. Smith, V. Narayanamurti, and J. E. Bowers, Microscale Thermophys. Eng. 2, 37 (1998). 